Universidad Nacional Experimental Francisco de Miranda (UNEFM). Santa Ana de Coro. Venezuela

Bayron Damián Loja-Gutama; Darwin Gabriel García-Herrera; Cristian Andrés Erazo-Álvarez; Juan Carlos Erazo-Álvarez

DOI 10.35381/cm.v6i3.395

\title{
Podcast como estrategia didáctica en la enseñanza de la expresión oral y escrita
}

Podcast as a didactic strategy in the teaching of oral and written expression

\author{
Bayron Damián Loja-Gutama \\ bayron.loja.52@est.ucacue.edu.ec \\ Universidad Católica de Cuenca, Azogues \\ Ecuador \\ https://orcid.org/0000-0002-3883-5156 \\ Darwin Gabriel García-Herrera \\ dggarciah@ucacue.edu.ec \\ Universidad Católica de Cuenca, Azogues \\ Ecuador \\ https://orcid.org/0000-0001-6813-8100 \\ Cristian Andrés Erazo-Álvarez \\ cristianerazo@ucacue.edu.ec \\ Universidad Católica de Cuenca, Cuenca \\ Ecuador \\ https://orcid.org/0000-0001-8746-4788 \\ Juan Carlos Erazo-Álvarez \\ jcerazo@ucacue.edu.ec \\ Universidad Católica de Cuenca, Cuenca \\ Ecuador \\ https://orcid.org/0000-0001-6480-2270
}

Recibido: 20 de agosto de 2020

Aprobado: 15 de noviembre de 2020 
Universidad Nacional Experimental Francisco de Miranda (UNEFM). Santa Ana de Coro. Venezuela

Bayron Damián Loja-Gutama; Darwin Gabriel García-Herrera; Cristian Andrés Erazo-Álvarez; Juan Carlos Erazo-Álvarez

\title{
RESUMEN
}

La presente investigación tiene la finalidad de implementar el podcast como estrategia didáctica en la enseñanza de la expresión oral y escrita, esto se debe a los problemas que año tras año se viene arrastrando desde los niveles iniciales de la institución. Metodológicamente fue de tipo descriptiva no experimental. Los resultados obtenidos revelaron que los docentes desconocen y no utilizan de manera permanente las TIC en su aula de clases y por ende desmotivan a los niños en la iniciación de la lectura como la escritura, por ello, la propuesta de incluir y motivar a los docentes en el uso del podcast como estrategia para mejorar la comunicación oral. En cuanto al uso del podcasting como opción metodológica, el docente debe tener la predisposición de innovar y buscar nuevas maneras de enseñar con el uso de las TIC.

Descriptores: Programa informático didáctico; tecnología educacional; tecnología de la información. (Palabras tomadas del Tesauro UNESCO).

\begin{abstract}
The present research aims to implement the podcast as a didactic strategy in the teaching of oral and written expression, this is due to the problems that year after year have been dragging from the initial levels of the institution. Methodologically, it was descriptive, not experimental. The results obtained revealed that teachers are unaware and do not permanently use ICT in their classroom and therefore demotivate children in the initiation of reading as well as writing, therefore, the proposal to include and motivate teachers in the use of the podcast as a strategy to improve oral communication. Regarding the use of podcasting as a methodological option, the teacher must have the predisposition to innovate and seek new ways of teaching with the use of ICT.
\end{abstract}

Descriptors: Educational software; educational technology; information technology. (Words taken from the UNESCO Thesaurus). 
Universidad Nacional Experimental Francisco de Miranda (UNEFM). Santa Ana de Coro. Venezuela

Bayron Damián Loja-Gutama; Darwin Gabriel García-Herrera; Cristian Andrés Erazo-Álvarez; Juan Carlos Erazo-Álvarez

\section{INTRODUCCIÓN}

La educación esta cambiando de una forma acelerada, donde los medios tecnológicos empiezan a tomar un rol muy importante dentro de la educación y con ello la docencia debe buscar nuevas maneras de enseñar a sus educandos en un mundo de constante cambio. Dentro de una sociedad globalizada, hiperconectada en la que vivimos, la comunicación juega un rol básico en el proceso de enseñanza - aprendizaje el correcto uso del feedback entre autores de una comunidad es esencial.

Diariamente observamos cambios e innovaciones tecnológicas, teniendo bastante incidencia en los procesos educativos con un impacto en la comunicación, si bien está crisis sanitaria es otro factor que suma una problemática para que el docente tenga que buscar nuevas alternativas o estrategias de enseñanza con los diferentes actores educativos; acomodarse y ser resilientes obliga a buscar otros mecanismos para desarrollar habilidades, destrezas y competencias en sus discentes. Los medios de comunicación hoy en día juegan un rol importante como la radio, la televisión y la web.

Las Tecnologías de Información y Comunicación [TIC] son herramientas innovadoras que han facilitado la interacción entre el profesor y el estudiante, afianzando de mejor manera el eje curricular integrador de Lengua y Literatura (Escuchar, hablar, leer y escribir para la interacción social) lo confirma (Ministerio Educación, 2016) en el currículo del área.

En Ecuador, todavía falta de difundir plataformas y aplicaciones en el uso de las TIC como herramienta innovadora de estudio, en el área de Lengua y Literatura. Sin embargo, el sistema educativo ha dado pasos importantes con programas educativos en el uso y manejo de las TIC, presentados desde el año 2009 para la aplicación de la tecnología dentro de las aulas en el proceso de enseñanza aprendizaje (Ministerio Educación, 2016). La palabra "podcasting" fue utilizado por primera vez en su artículo "Audible Revolution" la misma que fue publicado en The Guardian (Hammersley, 2004) donde mencionaba un "new boom in amateur radio" teniendo una expansión generalmente de sonido en formato 
Universidad Nacional Experimental Francisco de Miranda (UNEFM). Santa Ana de Coro. Venezuela

Bayron Damián Loja-Gutama; Darwin Gabriel García-Herrera; Cristian Andrés Erazo-Álvarez; Juan Carlos Erazo-Álvarez

MP3 u OGG. Hammersley comenzó a formular varios nombres como "audioblogging, guerrilla media o podcasting", quedando el último término como proyección.

A raíz de ello se busca llevar una experiencia innovadora con una dinamica de aprendizaje en donde los estudiantes puedan acceder al aprendizaje con ciertas habilidades digitales tales como saber usar una computadora, el celular, o hasta saber descargar documentos en línea. La enseñanza, se ve cada vez diferente, la práctica diaria del docente se debe volver más dinámica para guiar a los alumnos y establecer una serie de acciones con las diferentes aplicaciones o plataformas digitales. El resultado es pretender desarrollar en el estudiante un aprendizaje autónomo guiado por el docente para lograr nuevos conocimientos que sean llevado a resolver problemas de la vida diaria. La propuesta investigativa titulada -el podcast como herramienta de aprendizaje: estrategias para la enseñanza de lengua y literatura- en estudiantes de la Unidad Educativa Remigio Crespo Toral [UERCT], parroquia La Asunción se comenzó con observaciones pasivas y participativas de clases también del análisis de resultados de diferentes concursos -Spelling bee, Remigio orador y pruebas ser-; por último, encuestas a docentes que imparten clases a los estudiantes.

Basándose en las evidencias que los estudiantes tienen dificultades en la expresión oral en aspectos como el uso de muletillas, barbarismos, fluidez, persuasión, discursos y temor escénico. Englobando los aspectos que acabamos de dar hacen que tengan problemas de expresión oral.

Una vez identificado las problemáticas se propone en diseñar y aplicar un conjunto de estrategias que vayan de la mano con el uso de las tics; el podcast es una gran herramienta sonora para trabajar hoy en día con los jóvenes en tiempos de COVID - 19. El uso de esta aplicación por los docentes es de mucha importancia ya que permite implementar el Tele-schooling y educación a distancia, desde sus casas para trabajar con los estudiantes. 
Universidad Nacional Experimental Francisco de Miranda (UNEFM). Santa Ana de Coro. Venezuela

Bayron Damián Loja-Gutama; Darwin Gabriel García-Herrera; Cristian Andrés Erazo-Álvarez; Juan Carlos Erazo-Álvarez

El uso del podcast aporta confianza, innovación y es un recurso de fácil compresión al momento de captar información (Ruiz, 2017) favoreciendo la adquisición de nuevas habilidades y competencias orales, con la implementación de diferentes estrategias metodologicas que ofrecen nuevas oportunidades de aprendizaje para el desarrollo comunicacional.

Con estos antecedentes, lo que se pretende como objetivo es desarrollar un curso sobre el uso del Podcast para mejorar la expresión oral y escrita, en los estudiantes de la UERCT para el desarrollo de habilidades comunicativas. Como pregunta guía del estudio fue ¿de qué manera el uso de podcast educativo, contribuye en los procesos de aprendizaje en la Expresión oral y escrita? También la hipotesis planteada es: A mayor uso del podcast, mejor desarrollo de habilidades comunicativas en los estudiantes.

\section{Referencial teórico}

La comunicación es uno de los medios importantes que deben ser innovados en la actualidad, buscar nuevas estrategias que vayan de la mano con el uso de las Tecnologías de información y comunicación [TIC] en la educación, según (Goldman, 2018) el uso de la tecnología ha adquirido gran relevancia en el campo educativo como una herramienta facilitadora en el proceso de enseñanza, siendo el docente mediador y guía en el uso de las diferentes plataformas y aplicaciones.

Según, (Jaimes \& Callejas, 2009) "La Organización de las Naciones Unidas para la educación, la Ciencia y la Cultura [UNESCO] define las TIC como el conjunto de disciplinas científicas, tecnológicas, de ingeniería y técnicas de gestión utilizadas en el manejo y procesamiento de la información, sus aplicaciones, los computadores y su interacción con hombres, las máquinas y los contenidos asociados de carácter social, económico y cultural" (p. 64). 
Universidad Nacional Experimental Francisco de Miranda (UNEFM). Santa Ana de Coro. Venezuela

Bayron Damián Loja-Gutama; Darwin Gabriel García-Herrera; Cristian Andrés Erazo-Álvarez; Juan Carlos Erazo-Álvarez

En la actualidad existen diversos estudios acerca del uso del podcast en la educación como estrategia de enseñanza, para lo cual se realizó una revisión bibliográfica de excelentes trabajos por países de acuerdo con su contenido.

Dice (Sigüenza, Montánchez , \& Palta, 2018) en el Ecuador, hacen alusión a que los estudiantes y docentes estamos inmersos en una cultura de innovación y digitalización y por eso la importancia de innovar nuevas maneras para llegar a los estudiantes en el proceso de enseñanza - aprendizaje. Manifiestan que la tecnología no puede quedar exenta en la educación, los docentes debemos buscar nuevas maneras para comunicarnos con los estudiantes, en la actualidad se han incorporado en las universidades aulas virtuales y otras herramientas web 2.0 y una de ellas es el podcast. De manera introductoria hacen un muestreo y análisis del uso del podcast en la Universidad Central del Ecuador teniendo resultados de muy provechosos en la audición de programas radiofónicos, pero no así en el podcast. Buscar formas y nuevas estrategias es el reto para los educadores, llevar estas nuevas herramientas al aula es importante para que los jóvenes puedan aprender, mencionando que el material ellos puedan descargarlos y a la vez aprender en diferentes partes donde se encuentren.

Como especifica (Saborío, 2018) de Costa Rica; el podcasting como estrategia de comunicación llevada a la educación es una idea innovadora, la misma es aplicable para entornos virtuales. El docente debe ir a la vanguardia de los nuevos cambios que se va dando en la actualidad, buscar herramientas y estrategias que llamen al estudiante la atención; en el manejo del podcast también se puede trabajar el aprendizaje colaborativo, aprendizaje autónomo y sobre todo realizar un repaso constante del proceso enseñanza y aprendizaje de los temas específicos, es importante resaltar la versatilidad que brinda para trabajar la interculturalidad y ciudadanía; los estudiantes deben asumir el protagonismo de las producciones de trabajo; ahora para llegar a la meta el educador debe brindar y trabajar con herramientas o dispositivos tecnológicos. Para la elaboración 
Universidad Nacional Experimental Francisco de Miranda (UNEFM). Santa Ana de Coro. Venezuela

Bayron Damián Loja-Gutama; Darwin Gabriel García-Herrera; Cristian Andrés Erazo-Álvarez; Juan Carlos Erazo-Álvarez

de un podcast generalmente existen dos fases importantes que son: la planificación y la elaboración.

Al respecto, (Suárez, Vélez, \& Londoño, 2018) parafraseando a autores chilenos, consideran que la finalidad de mejorar la literacidad es trabajando con estrategias metacognitivas para fortalecer la comprensión lectora, la misma que debe ir acompañado del uso de las TIC, tal el caso es mediante la creación de videos, fotos y podcast. La literacidad va de la mano con la comprensión lectora para que la enseñanza se vuelva dinámica y didáctica enfocada en los estudiantes para promover el aprendizaje significativo. Lo que busca la literacidad es mejorar la comprensión lectora y aumentar el rendimiento académico volviéndose más críticos y reflexivos en el contexto que se desenvuelven diariamente.

(Cassany, 2007) expone que el docente es el mediador y guía del proceso enseñanza aprendizaje y debe motivar y desarrollar la escritura completa de textos es decir oraciones completas, mas que oraciones simples y cortas, ya que la actividad reforzaría de gran manera el proceso de aprehensión en la escritura. Por último, el podcast ha desarrollado en los estudiantes nuevas experiencias radiales con el objeto de mejorar la expresión oral, la literalidad y habilidades transversales mediante el uso de nuevas tecnologías.

\section{Las TIC en la educación.}

En las últimas décadas, la incorporación de las herramientas Tecnológicas de la información y la comunicación [TIC] ha revolucionado el mundo, cambiando y a la vez modificando los hábitos de las personas; pero de manera específica en la comunicación de los individuos al momento de dialogar o interactuar partiendo desde diferentes campos como: la industria, el comercio, la medicina, la ingeniería y sobre todo el cambio profundo en la educación.

La incorporación de estas tecnologías al aula promueve nuevas formas de aprendizaje, nos abre hacia un universo digital, que se puede realizar, a distancia y virtualmente como 
Universidad Nacional Experimental Francisco de Miranda (UNEFM). Santa Ana de Coro. Venezuela

Bayron Damián Loja-Gutama; Darwin Gabriel García-Herrera; Cristian Andrés Erazo-Álvarez; Juan Carlos Erazo-Álvarez

indica (Begoña, 2016) el educador debe incorporar recursos tecnológicos para promover nuevas formas de conocimiento, valores y actitudes que mejoren la sociedad globalizada para el desarrollo de una ciudadanía competente, buscando el progreso y no la desigualdad. La aparición y masificación de la web 2.0 y el uso de las Tics provocaron un nuevo cambio en el ecosistema de información, en donde los medios tradicionales que producían una cierta cantidad de información tenían que pasar a producir de una manera más acelerada y con menos recursos porque la sociedad lo demandaba buscando que se más «participativa», en la forma de transmitir y compartir información (Canavilhas, 2015).

Hoy en día las nuevas generaciones conviven con la tecnología, por lo tanto, estos recursos deben ser aprovechados al máximo en la educación y cultura porque las Tics ofrecen infinitas herramientas para ser aplicadas en la enseñanza educativa mejorando la calidad de aprendizaje, la creatividad, la innovación, curiosidad e investigación. Además de la tecnología es importante que el docente reflexione sobre su propia práctica, evaluando cada una de las herramientas y de qué manera contribuyen estos recursos en el proceso de enseñanza - aprendizaje.

Las herramientas tecnológicas en la educación abarcan un conjunto de tecnologías que han venido para quedarse por su protagonismo en el ecosistema educativo, convirtiéndose en instrumentos digitales para los procesos educativos y formativos. Las tecnologías multimedia e internet son muy versátiles al momento de interactuar, despertando en cada estudiante expectativas de nuevas formas de aprendizaje. El uso del podcast en la educación fomenta el desarrollo de habilidades lectoras y verbales, es uno de los primeros pasos para integrar las TIC en el aula, instaurando así una experiencia de aprendizaje dinámica realizando un intercambio de información constante. Conociendo que el uso de podcast es una gran herramienta didáctica para el aprendizaje y desarrollo de habilidades en Lengua y Literatura es factible que cada docente realice su propio podcast, enfocándolos a sus contextos de enseñanza, al público dirigido y sobre 
Universidad Nacional Experimental Francisco de Miranda (UNEFM). Santa Ana de Coro. Venezuela

Bayron Damián Loja-Gutama; Darwin Gabriel García-Herrera; Cristian Andrés Erazo-Álvarez; Juan Carlos Erazo-Álvarez

todo cubrir el objetivo de aprendizaje, procurando que el discente desarrolle aprendizajes constructivos de una manera autónoma es decir una educación asincrónica (Peralta, 2016). Además, el podcast es un recurso que puede ser escuchado o descargado de la web en el horario que el radioescucha lo requiera desde cualquier dispositivo móvil.

\section{Aprendizaje móvil.}

En los últimos años la incorporación de las TIC en la educación, ha adquirido una creciente importancia y evolución colmado de actividades innovadoras de carácter colaborativo convirtiendo al estudiante en el constructor de su propio conocimiento con el docente como un guía y mentor, dándole la libertad necesaria para que explore el ecosistema tecnológico.

El aprendizaje móvil o mobile learning es el proceso de enseñanza que se ejecuta a través de los dispositivos móviles (teléfonos celulares, tabletas informáticas, etc.) conectados a una red inalámbrica dando una interacción inmediata entre docente y estudiante. Para los autores (Bukharaev \& Wisam, 2017) la educación móvil es una nueva forma de aprendizaje que tiene muchos beneficios educativos, en donde el discente puede interactuar con sus compañeros y profesores de una forma inmediata, facilitando el proceso de aprendizaje. Las barreras de conectividad, el acceso y la velocidad a la información son factores muy importantes y a la vez asequibles para cada estudiante por su costo y versatilidad de poder consultar información por un dispositivo móvil favoreciendo a los autores en la enseñanza y el aprendizaje. 
Universidad Nacional Experimental Francisco de Miranda (UNEFM). Santa Ana de Coro. Venezuela

Bayron Damián Loja-Gutama; Darwin Gabriel García-Herrera; Cristian Andrés Erazo-Álvarez; Juan Carlos Erazo-Álvarez

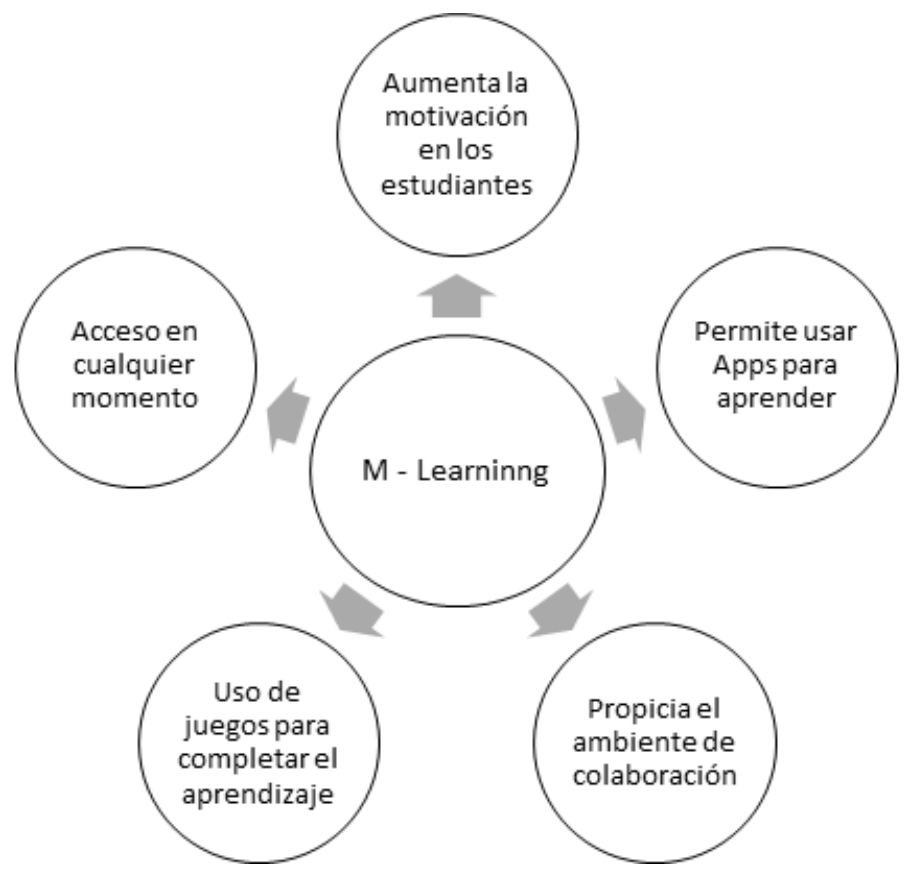

Figura 1: Proceso de planificación $M$ - Learninng.

Fuente: Adaptado de evirtualplus.

Para la (UNESCO, 2013) el aprendizaje móvil es parte de las TIC en la educación. La tecnología es más accesible y de fácil adquisición propiciando la aparición de nuevos dispositivos móviles y a la vez nuevas dinámicas de interacción entre docente y estudiantes para favorecer nuevas prácticas colaborativas en el aprendizaje de las personas en cualquier momento y lugar.

\section{Podcast y su elaboración}

Es importante empezar hablando desde el origen de las cosas, para lo cual vamos a definir un Podcast; según (Hsiao, 2015) indica que surge de un término acrónimo, la combinación de pod (cápsula) y broadcast (difusión-emisión) son archivos de audios en diferentes formatos como audioblogs, bitácoras o weblogs; producidos por uno o varios autores para ser publicados y reproducidos en diferentes dispositivos. Después de haber realizado una revisión documental que se ha realizado de antecedente, la misma que fue 
Bayron Damián Loja-Gutama; Darwin Gabriel García-Herrera; Cristian Andrés Erazo-Álvarez; Juan Carlos Erazo-Álvarez

presentada de diferentes tesis, artículos y revistas que me han ido dando pautas de que una educación interactiva con el uso de las TIC si podemos encajar en la educación.

A su vez (Solano \& Sanchez, 2010) en las instituciones educativas para la realización de un podcast educativo es importante seguir un proceso de planificación, para lo cual es recomendable considerar los siguientes aspectos:

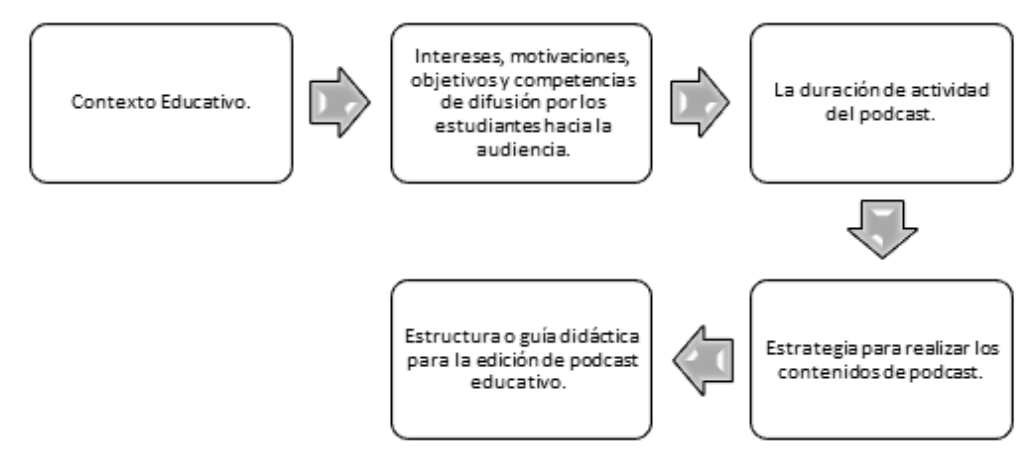

Figura 2: Proceso de planificación.

Fuente: Adaptado de Solano y Sánchez (2010).

Desde la perspectiva de (Robles, 2010) con el procedimiento de la aplicación en el aula, manifiesta que los podcasts fueron planificados con su grupo para 15 minutos de una manera secuencial y significativo para sus discentes. Desarrollando siete podcasts desde las necesidades que pudo observar en el curso. 
Universidad Nacional Experimental Francisco de Miranda (UNEFM). Santa Ana de Coro. Venezuela

Bayron Damián Loja-Gutama; Darwin Gabriel García-Herrera; Cristian Andrés Erazo-Álvarez; Juan Carlos Erazo-Álvarez

De esta manera se describe el proceso de los podcasts:

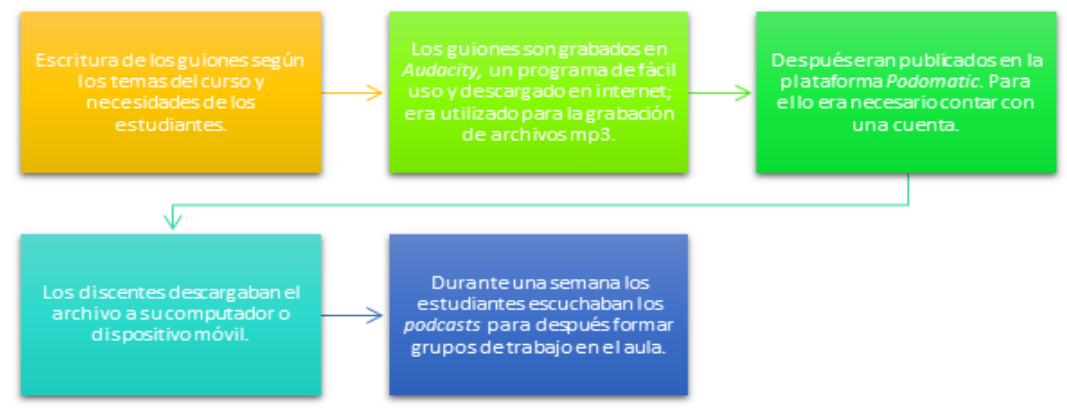

Figura 3: Proceso de elaboración.

Fuente: Adaptado de Robles (2010).

\section{METODOLOGÍA}

El proceso metodológico de la presente investigación es de carácter no experimental transversal, con el análisis respectivo de las variables mediante el cálculo de Chicuadrado de Pearson y frecuencia, con el propósito de conocer la asociación de las diferentes variables de estudio (Carreño-Godoy, Erazo-Álvarez, Narváez-Zurita, \& Moreno, 2020), para lo cual se da apertura a nuevas líneas de investigación llevando a la realidad y contexto de cada participante en el proceso de investigación, mediante la plataforma de Microsoft Forms a través de una encuesta online y cuestionario de diferentes alternativas basadas en la escala de Likert que según (Ospina, Sandoval, Aristizábal, \& Ramírez, 2005), es importante para identificar actitudes, ya que permite alcanzar niveles de confiabilidad en la investigación.

Para la recolección de datos se utilizó la técnica de la encuesta, misma que permite recoger información de la población de estudio, se aplicó la encuesta a un total de 24 docentes de la Unidad Educativa Remigio Crespo Toral desde Inicial a Bachillerato, para conocer las formas de enseñanza y estrategia con el uso de las Tic que tienen en la 
Bayron Damián Loja-Gutama; Darwin Gabriel García-Herrera; Cristian Andrés Erazo-Álvarez; Juan Carlos Erazo-Álvarez

expresión oral y escrita de las asignaturas. El instrumento fue validado mediante el coeficiente alfa de Cronbach con un valor de 0,914, cuyo procesamiento de información se realizó con la ayuda del software estadístico SPSS en su versión 19 para el debido análisis, interpretación, comparación de variables y confirmación de la hipótesis; el cual nos dará una perspectiva para identificar el problema y así llegar a una fundamentación llevándonos a terminar con el presente trabajo.

\section{RESULTADOS}

Para empezar con el análisis de la presente investigación se realizó la aplicación de encuestas. De las variables se hizo un análisis de normalidad de las variables, las mismas que me permiten dar una respuesta al objetivo de investigación. A continuación, se muestran las siguientes tablas con la síntesis de los resultados obtenidos:

\section{Tabla 1.}

Frecuencia de uso del podcast.

\begin{tabular}{cccccc}
\hline & & Frecuencia & Porcentaje & Porcentaje válido & $\begin{array}{c}\text { Porcentaje } \\
\text { acumulado }\end{array}$ \\
\hline \multirow{4}{*}{ Válidos } & Siempre & 5 & 20.8 & 20.8 & 20.8 \\
& Casi siempre & 1 & 4.2 & 4.2 & 25.0 \\
& A veces & 3 & 12.5 & 12.5 & 37.5 \\
& Rara vez & 3 & 12.5 & 12.5 & 50.0 \\
& Nunca & 12 & 50.0 & 50.0 & 100.0 \\
\cline { 2 - 6 } & Total & 24 & 100.0 & 100.0 & \\
\hline
\end{tabular}

Fuente: Elaboración propia. 
Bayron Damián Loja-Gutama; Darwin Gabriel García-Herrera; Cristian Andrés Erazo-Álvarez; Juan Carlos Erazo-Álvarez

En la tabla 1 se observa que el 50\% nunca utiliza el podcast, razón por la cuál es necesario fortalecer su empleo en el ámbito educativo a través de una capacitación permanente. La investigación demostró que del $100 \%$ de los encuestados, en su gran mayoría desconoce la herramienta tecnológica del podcast.

Tabla 2.

Utilización de las Tics en el aula.

\begin{tabular}{cccccc}
\hline & & Frecuencia & Porcentaje & Porcentaje válido & $\begin{array}{c}\text { Porcentaje } \\
\text { acumulado }\end{array}$ \\
\hline \multirow{4}{*}{ Válidos } & Rara vez & 3 & 12.5 & 12.5 & 12.5 \\
& A veces & 3 & 12.5 & 12.5 & 25.0 \\
& Casi siempre & 11 & 45.8 & 45.8 & 70.8 \\
& Siempre & 7 & 29.2 & 29.2 & 100.0 \\
\cline { 2 - 6 } & Total & 24 & 100.0 & 100.0 & \\
\hline
\end{tabular}

Fuente: Elaboración propia.

En cuanto a los resultados en torno a la utilización de las Tic en el aula el 70,8\% casi siempre utiliza las Tic en el aula, mientras que el $29,2 \%$ siempre utiliza las Tics en el aula. Lo cual nos demuestra que los docentes vienen aplicando las Tics al momento de impartir clases. 
CIENCIAMATRIA

Revista Interdisciplinaria de Humanidades, Educación, Ciencia y Tecnología

Año VI. Vol. VI. N³. Edición Especial III. 2020

Hecho el depósito de ley: pp201602FA4721

ISSN-L: 2542-3029; ISSN: 2610-802X

Universidad Nacional Experimental Francisco de Miranda (UNEFM). Santa Ana de Coro. Venezuela

Bayron Damián Loja-Gutama; Darwin Gabriel García-Herrera; Cristian Andrés Erazo-Álvarez; Juan Carlos Erazo-Álvarez

Tabla 3.

Frecuencia del uso de estrategias de enseñanza en la Expresión Oral y Escrita.

\begin{tabular}{cccccc}
\hline & & Frecuencia & Porcentaje & Porcentaje válido & $\begin{array}{c}\text { Porcentaje } \\
\text { acumulado }\end{array}$ \\
\hline \multirow{7}{*}{ Válidos } & Nunca & 10 & 41.7 & 41.7 & 41.7 \\
& Rara vez & 4 & 16.7 & 16.7 & 58.3 \\
& A veces & 3 & 12.5 & 12.5 & 70.8 \\
& Casi siempre & 3 & 12.5 & 12.5 & 83.3 \\
& Siempre & 4 & 16.7 & 16.7 & 100.0 \\
\cline { 2 - 6 } & Total & 24 & 100.0 & 100.0 & \\
\hline
\end{tabular}

Fuente: Elaboración propia.

Con respecto a la información si los docentes usan estrategias de enseñanza en la Expresión oral y Escrita mediante el uso de las Tic, la tabla 3 evidencia que el 41,7\% nunca utiliza, razón por la cual es necesario fortalecer las estrategias de enseñanza en la expresión oral y escrita para fortalecer la literalidad.

\section{Tabla 4}

Frecuencia del uso de las Tics en el mejoramiento de la Expresión Oral y Escrita

\begin{tabular}{cccccc}
\hline & & Frecuencia & Porcentaje & Porcentaje válido & $\begin{array}{c}\text { Porcentaje } \\
\text { acumulado }\end{array}$ \\
\hline \multirow{4}{*}{ Válidos } & Rara vez & 1 & 4.2 & 4.2 & 4.2 \\
& A veces & 6 & 25.0 & 25.0 & 29.2 \\
& Casi siempre & 11 & 45.8 & 45.8 & 75.0 \\
& Siempre & 6 & 25.0 & 25.0 & 100.0 \\
\cline { 2 - 6 } & Total & 24 & 100.0 & 100.0 & \\
\hline
\end{tabular}

Fuente: Elaboración propia. 
CIENCIAMATRIA

Revista Interdisciplinaria de Humanidades, Educación, Ciencia y Tecnología

Año VI. Vol. VI. N³. Edición Especial III. 2020

Hecho el depósito de ley: pp201602FA4721

ISSN-L: 2542-3029; ISSN: 2610-802X

Universidad Nacional Experimental Francisco de Miranda (UNEFM). Santa Ana de Coro. Venezuela

Bayron Damián Loja-Gutama; Darwin Gabriel García-Herrera; Cristian Andrés Erazo-Álvarez; Juan Carlos Erazo-Álvarez

En la tabla 4 se puede determinar que el uso de las Tics en el mejoramiento de la Expresión Oral y Escrita con el 4.2\% los docentes rara vez lo utilizan. Esto nos permite identificar que los docentes no están utilizando las Tics para mejorar la Expresión Oral y Escrita.

De esta manera se evidencia que el personal docente de la UERCT no está utilizando las Tics y estrategias en la expresión oral y escrita.

\section{Tabla 5.}

Tabla de contingencia Uso del Podcast. * Desarrolla habilidades de aprendizaje en la Expresión Oral y Escrita.

\begin{tabular}{|c|c|c|c|c|c|c|}
\hline & & \multicolumn{4}{|c|}{ Desarrolla habilidades de aprendizaje en la Expresión Oral y Escrita. } & \multirow{2}{*}{ Total } \\
\hline & & Rara vez & A veces & Casi siempre & Siempre & \\
\hline \multirow{5}{*}{ Uso del Podcast. } & Siempre & 0 & 0 & 4 & 1 & 5 \\
\hline & Casi siempre & 0 & 0 & 1 & 0 & 1 \\
\hline & A veces & 0 & 0 & 1 & 2 & 3 \\
\hline & Rara vez & 0 & 1 & 2 & 0 & 3 \\
\hline & Nunca & 1 & 3 & 5 & 3 & 12 \\
\hline \multicolumn{2}{|c|}{ Total } & 1 & 4 & 13 & 6 & 24 \\
\hline \multicolumn{7}{|c|}{ Pruebas de chi-cuadrado } \\
\hline & & \multicolumn{2}{|r|}{ Valor } & gl & \multicolumn{2}{|c|}{ Sig. asintótica (bilateral) } \\
\hline \multicolumn{2}{|c|}{ Chi-cuadrado de Pearson } & \multicolumn{2}{|r|}{$8.310^{\mathrm{a}}$} & 12 & \multicolumn{2}{|c|}{.760} \\
\hline \multicolumn{2}{|c|}{ Razón de verosimilitudes } & \multicolumn{2}{|r|}{10.264} & 12 & \multicolumn{2}{|c|}{.593} \\
\hline \multicolumn{2}{|c|}{ Asociación lineal por lineal } & \multicolumn{2}{|r|}{1.313} & 1 & \multicolumn{2}{|c|}{.252} \\
\hline \multicolumn{2}{|c|}{$\mathrm{N}$ de casos válidos } & \multicolumn{2}{|r|}{24} & & & \\
\hline
\end{tabular}

a. 19 casillas (95.0\%) tienen una frecuencia esperada inferior a 5. La frecuencia mínima esperada es .04.

Fuente: Elaboración propia. 
Universidad Nacional Experimental Francisco de Miranda (UNEFM). Santa Ana de Coro. Venezuela

Bayron Damián Loja-Gutama; Darwin Gabriel García-Herrera; Cristian Andrés Erazo-Álvarez; Juan Carlos Erazo-Álvarez

Al realizar la comparación entre las variables, el uso del podcast y desarrollo de habilidades de aprendizaje en la expresión oral y escrita, se obtuvo un valor de 0,760 determinando de está una hipótesis nula es decir que no existe relación entre el podcast y el desarrollo de habilidades de aprendizaje. De esta manera se hace hincapié en que los docentes no utilizan el podcast para desarrollar destrezas de expresión oral y escrita, ver tabla 5.

En general en base a los resultados podemos mencionar la mayoría de los docentes de la institución desconocen el uso del podcast y estrategias para el desarrollo de la expresión oral y escrita. De la misma manera como aspecto positivo podemos señalar la utilización de las TIC por la mayoría de los profesores con sus estudiantes en las aulas, dando luz verde para implementar el uso del podcast en los estudiantes de la UERCT.

El podcast como estrategia para mejorar la expresión oral y escrita será una gran herramienta que permitirá al discente experimentar nuevas formas de aprendizaje llevando a una retroalimentación, un intercambio de ideas y proporcionando nuevos conocimientos para conseguir un aprendizaje y autoaprendizaje eficaz.

Es por ello que se concuerda con un aporte relevante de (Phillips, 2017) afirma que los beneficios del podcast en el aprendizaje son adecuados para los estudiantes brindándoles mayor lenguaje en la fluidez y adquisición de vocabulario, también asevera que las actividades de podcasting son adecuadas para estudiantes que suelen estar callados en clases debido a la falta de fluidez y la mala pronunciación, ya sea por problemas psicológicos (vergüenza, estrés, temor y pánico) al hablar en público, económicos (no disponibilidad del material), familiares, $u$ otras causas que tienen que ver con el hecho de expresarse adecuadamente.

Tras lecturas de algunos autores se confirma que aprender y enseñar con podcasts, tiene grandes beneficios y más aun con estrategias didácticas enfocadas en mejorar el aprendizaje y autoaprendizaje en los estudiantes; de allí surge nuestro interés por indagar nuevas formas para enseñar la oralidad y escritura en los estudiantes. Es necesario 
Universidad Nacional Experimental Francisco de Miranda (UNEFM). Santa Ana de Coro. Venezuela

Bayron Damián Loja-Gutama; Darwin Gabriel García-Herrera; Cristian Andrés Erazo-Álvarez; Juan Carlos Erazo-Álvarez

considerar lo planteado (Peralta, 2016)quien menciona que, para fomentar la motivación en el aula, es necesario trabajar con recursos sincrónicos y asincrónicos desarrollando mejores habilidades en la expresión oral y escrita porque les brinda la oportunidad de llevar su propio ritmo de trabajo sin el estrés de interacción en tiempo real.

\section{PROPUESTA}

En el presente trabajo empiezo haciendo una reflexión del ¿Por qué la necesidad de innovar en educación? La respuesta es porque en el aprendizaje la innovación es una acción que transforma y genera un profundo cambio en un sistema educativo imperfecto, la realidad es que el sistema educativo ecuatoriano presenta y adolece de una serie de problemas y fallos que son descubiertos en las diferentes evaluaciones realizadas por el Instituto de Evaluación [INEVAL] a los estudiantes de las diferentes instituciones educativas del país.

La inclusión de nuevas tecnologías en el aula, nos brindara una mayor motivación por parte del alumnado en desarrollar el trabajo colaborativo, cooperativo y autónomo para mejorar habilidades, destrezas y competencias.

Como acabamos de adelantar en lo expuesto y en base a los objetivos y encuestas, se presenta una propuesta para motivar a los docentes el manejo y uso de las TIC, en el desarrollo de la expresión oral y escrita. El podcast surge como propuesta para generar aulas sin muros, al alcance de todos para crear y distribuir contenidos educativos apropiados al entorno de aprendizaje con estrategias para el desarrollo de la expresión oral y escrita en las diferentes asignaturas el cual permitirá trabajar diferentes Macro destrezas para cumplir con uno de los objetivos que pide el currículo nacional ecuatoriano. A continuación, les presento el modelo y los pasos a seguir para su correcta implementación del podcast en los diferentes establecimientos educativos. 
Bayron Damián Loja-Gutama; Darwin Gabriel García-Herrera; Cristian Andrés Erazo-Álvarez; Juan Carlos Erazo-Álvarez

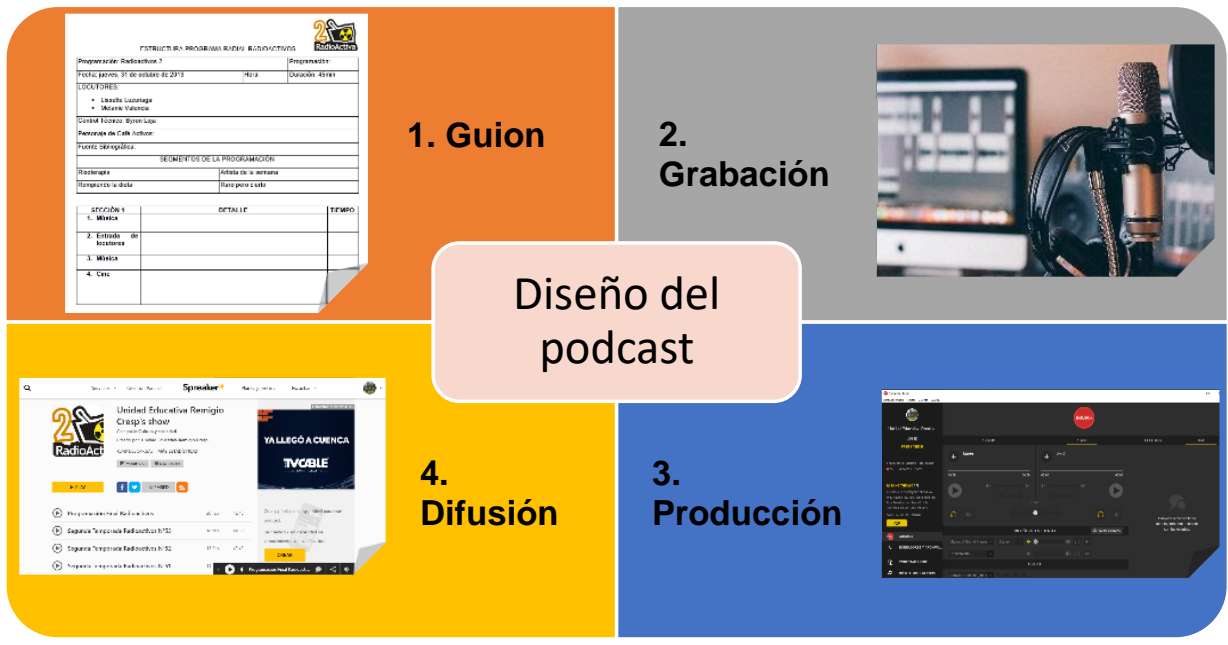

Figura 4. Pasos básicos para la realización de un podcast.

Fuente: Elaboración propia.

En el gráfico podemos observar los cuatro pasos para diseñar un podcast, el orden a realizar es:

1. Diseñar el guion.

2. Grabar el programa.

3. Producción y edición del guion.

4. Difusión del podcast.

Empezaremos indicando que el podcast no es un archivo único, más bien se trata de una actualización continua de archivos. El leguaje radiofónico en donde podemos encontrar la voz del locutor, la música, algunos efectos sonoros entre otros son componentes del lenguaje que desempeñan algunas funciones: sintonía, programación, cortes musicales dándole una atmosfera determinada que acompañe la voz del locutor.

Paso 1: Elaborar un guion, para comenzar con el primer paso -diseño del guion- debemos tener en cuenta el material que pretendemos incluir en el aula, analizar si realmente es adecuado para la hora clase, ver sus características, motivaciones, intereses y sobre todo el procedimiento metodológico con sus respectivas estrategias para la producción de 
Universidad Nacional Experimental Francisco de Miranda (UNEFM). Santa Ana de Coro. Venezuela

Bayron Damián Loja-Gutama; Darwin Gabriel García-Herrera; Cristian Andrés Erazo-Álvarez; Juan Carlos Erazo-Álvarez

contenidos bajo una estructura didáctica, es decir una ficha para la edición de podcast educativo que a continuación se la presenta (figura 5). La misma hace énfasis a ser sencilla y didáctica su esquema para la comprensión de los estudiantes.

Los podcasts que tendrán que ser elaborados por los estudiantes serán cortos entre 15 a 25 minutos, es importante seguir una secuencia de manera organizada donde la primera actividad es crear un guion, ya con un tema establecido y la información necesaria, es importante que exista investigación, retroalimentación y corrección del guion producido.

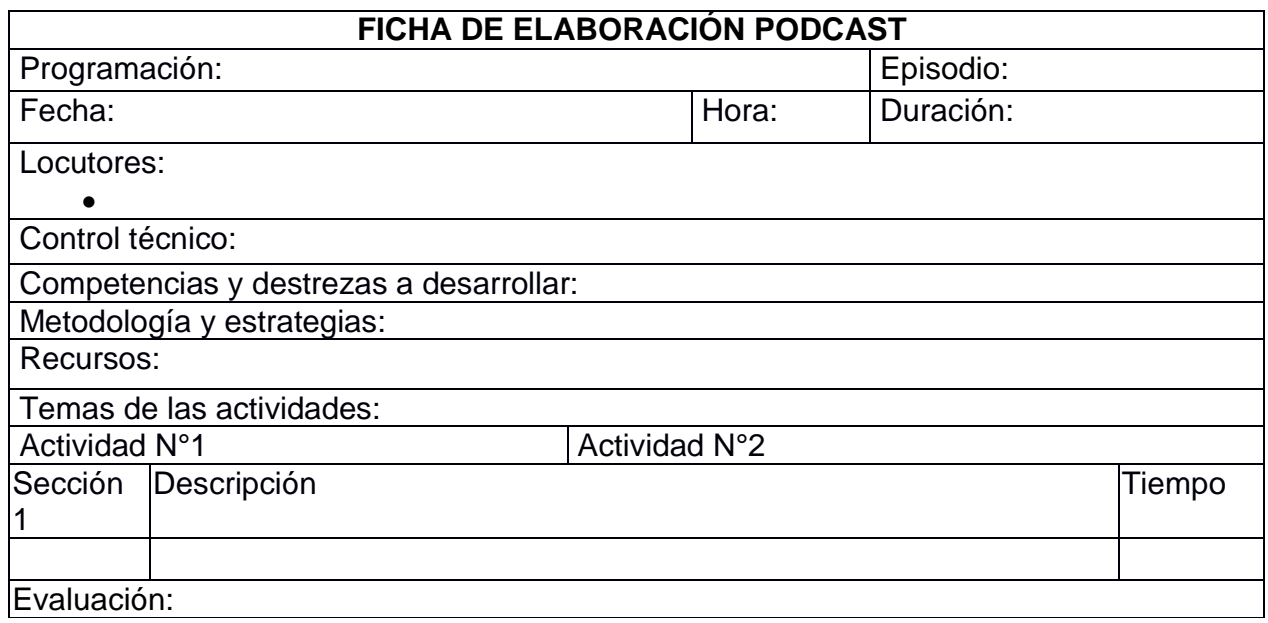

Figura 5. Ficha para la edición de podcast educativo.

Fuente: Elaboración propia.

Paso 2: Grabación del programa, en el presente paso es importante seleccionar el software que se va utilizar. En el mercado digital existe una amplia variedad de aplicaciones para crear y escuchar podcast, en particular se recomienda la aplicación Spreaker, es una aplicación de fácil uso y con alta calidad. La aplicación móvil para iOS y Android ofrece variedades de recursos en donde pueden crear, albergar y distribuir el contenido de audio con facilidad. Además, incluye una consola para grabar, espacio para almacenar temas musicales, acceso a redes sociales y otros beneficios más. 
Universidad Nacional Experimental Francisco de Miranda (UNEFM). Santa Ana de Coro. Venezuela

Bayron Damián Loja-Gutama; Darwin Gabriel García-Herrera; Cristian Andrés Erazo-Álvarez; Juan Carlos Erazo-Álvarez

Podemos ir al sitio web oficial para descargar la aplicación en el escritorio o en su móvil, la dirección web es: http://www.spreaker.com

Para empezar con la elaboración del podcast es importante plantearse incluir en el aula como herramienta digital y a la vez adecuar para nuestra clase, como también el desarrollo de contenidos si van a ser semanales, quincenales o mensuales. Ahora el docente debe tener en cuenta la audiencia, sus estudiantes viendo los intereses y motivaciones para posteriormente desarrollar el contenido con el material correcto.

Sin embargo, el docente debe ya poseer algunas estrategias para empezar a trabajar la expresión oral y escrita, vamos a detallar algunas de ellas:

Aprendizaje cooperativo: Brindándole al docente el rol de diseñador y mediador de tareas bajo una estrategia didáctica enfocada a las actividades grupales que promueva la interacción entre los discentes, la participación activa, el intercambio de ideas, la empatía y sobre todo el respeto, bajo todas estas expectativas que podemos llevar al aula en donde podamos alcanzar los objetivos de mejorar la lectura y escritura, teniendo la interacción y el desarrollo de actividades de forma autónoma para la presentación de contenidos de manera grupal a través del podcast.

Aprendizaje basado en proyectos (ABP): En la actualidad la enseñanza - aprendizaje basada en proyectos es un método educativo que busca trabajar en equipo con un objetivo definido, asignando roles y propiciando la autonomía para trabajar en grupos. Dentro de estos equipos que se llegan a conformar podemos ver las relaciones y las formas de interactuar, teniendo una libertad de opinión, un buen trato y sobre todo respetado la idea del compañero. Ahora bien, al llevar está metodología con el uso del podcast, podemos desarrollar de manera individual y grupal competencias comunicativas en donde, el discente elabora su contenido buscando estrategias para la elaboración del podcast en conclusión afianza contenidos, mejora su oralidad y su escritura por el feedback que existe en el grupo estudiantil. 
Universidad Nacional Experimental Francisco de Miranda (UNEFM). Santa Ana de Coro. Venezuela

Bayron Damián Loja-Gutama; Darwin Gabriel García-Herrera; Cristian Andrés Erazo-Álvarez; Juan Carlos Erazo-Álvarez

Libro auditivo: La lectura debe ser vista como un proceso interactivo, la tarea de leer no podemos aislar a la actividad de escribir. La modalidad es transformar la experiencia lectora en una auditiva, mediante el uso de efectos de sonido, música y otros elementos auditivos. La estrategia de grabarse la lectura de un documento para luego ser escuchada tiene bastantes beneficios como detectando por el propio estudiante su tonalidad, entonación y errores u omisiones de fonemas o palabras. Trabajar está estrategia llevara al estudiantado a mejorar la literalidad.

Programa de entrevista: Es considerada como una estrategia comunicativa que fortalece la expresión oral y escrita, una de las principales ventajas es la manera de evaluar la comprensión, ya que constituye una conversación personalizada ayudando a perder el miedo escénico, los nervios, la tartamudez y sobre todo a tener mayor confianza al momento de dialogar. El podcast se convierte en un buen aliado como herramienta para las transmisiones de entrevista ya sea de manera online como offline.

Juegos de palabras: Una de las estrategias más animadas que desarrolla las capacidades del pensamiento y la creatividad. Dentro de los juegos de palabras como: anagramas, adivinanzas, chistes, retahílas, refranes, entre otros, se aprovechan para trabajar características lingüísticas y lograr mejores estructuras dando como resultado una mejor inteligencia verbal para la comprensión lectora.

La mayoría de estas actividades pueden ser diseñadas por los profesores y estudiantes al momento de elaborar su guion para realizar la grabación del podcast.

Paso 3: Producción o montaje, una vez ya elaborado la grabación es necesario realizar algunos ajustes a tener en cuenta como: agregar música de fondo, efectos de sonido, regrabar, borrar entre otras opciones más. Al momento de crear el podcast debemos considerar algunos aspectos técnicos que anteriormente ya lo mencionamos, es importante tener en cuenta el formato que se utiliza, con frecuencia y en su gran mayoría es el MP3, aunque no podemos soslayar otros formatos como ACC, OGG y WMA; son 
Universidad Nacional Experimental Francisco de Miranda (UNEFM). Santa Ana de Coro. Venezuela

Bayron Damián Loja-Gutama; Darwin Gabriel García-Herrera; Cristian Andrés Erazo-Álvarez; Juan Carlos Erazo-Álvarez

otros tipos de audio que influye la calidad del archivo. Mientras más variaciones de audio se ofrezca a las personas, se obtendrá una mayor cantidad de oyentes. Lo importante de todo es que puede ser descargado por los estudiantes desde un móvil para ser utilizado en sus hogares, en clases o en cualquier momento que crea conveniente.

Paso 4: Difusión del podcast, para el último paso es importante disponer de internet para así subirlo a la web del programa que haya adquirido; el audio debe ser guardado y a la vez identificado mediante un feed y metadatos. Tanto el feed como los metadatos permitirán organizar de una mejor manera los podcasts ya que podemos numerar por capítulos y describir cada uno de ellos llevando una biblioteca de manera organizada y secuenciada. Si se desea que nuestros podcasts tengan un mayor alcance a otros sectores es importante difundir mediante las redes sociales como: Facebook, Instagram, Twitter y WhatsApp. De la misma manera, como todo proceso, es importante retroalimentar mediante la lectura de los comentarios que hagan en los blogs, páginas web o foros que disponga el creador del podcast.

\section{CONCLUSIONES}

El presente trabajo concluye con tres valiosas aportaciones sobre el podcast educativo: Como primera aportación podemos decir, que los podcasts educativos crean ambientes constructivistas mediante entornos virtuales de aprendizaje que generan y desarrollan habilidades de lectura y escritura sobre todo lingüísticas. La integración de nuevas estrategias conjuntamente con el uso de las TIC conduce a resultados muy positivos. EI podcast brinda medios sonoros, que ofrecen gran cantidad de posibilidades y mecanismos de reproducción portátiles de mp3 los mimos que son de fácil portabilidad al momento de descargar de la web.

En cuanto al uso del podcasting como opción metodológica, el docente debe tener la predisposición de innovar y buscar nuevas maneras de enseñar con el uso de las TIC y 
Universidad Nacional Experimental Francisco de Miranda (UNEFM). Santa Ana de Coro. Venezuela

Bayron Damián Loja-Gutama; Darwin Gabriel García-Herrera; Cristian Andrés Erazo-Álvarez; Juan Carlos Erazo-Álvarez

una de ellas es el manejo del podcast para desarrollar nuevas formas de aprendizaje en sus estudiantes. Es importante conocer y dar a conocer el funcionamiento del podcast, el mismo que permitirá profundizar las habilidades cognitivas y contenidos señalados en un proceso de enseñanza y aprendizaje con una metodología dinámica e interactiva que aumente los niveles de motivación y el aprendizaje autónomo y significativo, brindando una flexibilidad al estudiante que genere su propia información con la interacción en las redes sociales.

Y como ultima conclusión, podemos manifestar que las potencialidades que brinda el podcast son numerosas, la implementación de estas nuevas formas y maneras de enseñar en las aulas con lleva mucha responsabilidad por parte de los docentes ya que involucra a que tengan que aprender a manejar estas nuevas herramientas tecnológicas, las mismas que deben ser aplicadas a contextos educativos dependiendo de los objetivos, y realidades que se encuentren los estudiantes. Las tecnologías, tales como el podcasting, pueden ayudar de manera significativa en la calidad de la educación, siempre y cuando el profesor y los estudiantes alcancen las metas y objetivos educativos que demanda el currículo nacional.

\section{REFERENCIAS}

Begoña, S. (2016). Las Tic y la educación social en el siglo XXI [ICT and social education in the twenty - first century]. Educación Mediatica y TIC, 8-24. Recuperado de https://n9.cl/bjhov

Bukharaev, N., \& Wisam, A. (25 de 09 de 2017). Mobile Learning Education has Become More Accessible. [La educación de aprendizaje móvil se ha vuelto más accesible]. ResearchGate, 5(2), 1-5. Recuperado de https://n9.cl/kdm9

Canavilhas, J. (2015). Nuevos medios, nuevo ecosistema. El profesional de la información, 24(4), 2-7. Recuperado de https://n9.cl/uprlt 
Universidad Nacional Experimental Francisco de Miranda (UNEFM). Santa Ana de Coro. Venezuela

Bayron Damián Loja-Gutama; Darwin Gabriel García-Herrera; Cristian Andrés Erazo-Álvarez; Juan Carlos Erazo-Álvarez

Carreño-Godoy, M., Erazo-Álvarez, J., Narváez-Zurita, C., \& Moreno, V. (2020). La responsabilidad social en las empresas camaroneras. [Social responsibility in shrimp companies]. Koinonia, 10, 1-29. doi:http://dx.doi.org/10.35381/r.k.v5i10.702

Cassany, D. (2007). Afilar el lapicero. Guía de redacción para profesionales. [Sharpen the pencil. Writing guide for porfessionals]. Barcelona: Universidad de Trento. Recuperado de https://n9.cl/oqop4

Goldman, T. (2018). The Impact of Podcasts in Education. [El impacto del Podcasts en la Educación]. Scholar Commons, 1-16. Recuperado de https://n9.cl/enwc1

Hammersley, B. (2004). Audible revolution. [Revolución audible]. The Guardian. Recuperado de https://n9.cl/vzc3k

Hsiao, D.-L. (2015). Efectos de distintos tipos de podcast en la fluidez oral de estudiantes. [Effects of different types of podcast on the oral fluency of students.]. Barcelona: Universitat de Barcelona. Recuperado de https://n9.cl/ofdw

Jaimes, G., \& Callejas, M. (2009). La autonomía, los procesos del pensamiento y las TIC : competencias del siglo XXI, temática contemporánea de aplicación en escuelas, colegios y universidades. [Autonomy, thought processes and TIC: 21st century competences, contemporary themes of app]. (Universidad Pedagógica y Tecnológica de Colombia, Ed.) Bogotá: Limusa. Recuperado de https://n9.cl/z4shh

Ministerio Educación. (2016). Currículo de los niveles de educación obligatoria. Quito: Ministerio de Educación. Recuperado de https://n9.cl/mnlj

Ospina, B., Sandoval, J., Aristizábal, C., \& Ramírez, M. (2005). La escala de Likert en la valoración de los conocimientos y las actitudes de los profesionales de enfermería en el cuidado de la salud. [The Likert scale in evaluathing the knowledge and attitudes of nursing professionals in health care]. Investigación y educación en enfermería, 13(1), 14-29. Recuperado de https://n9.cl/di0v

Peralta, O. (2016). Uso de podcats en entornos virtuales de aprendizaje para el desarrollo de habilidades linguisticas del idioma inglés. [Use of podcats in virtual learning environments for the development of English language skills.]. Puebla: Universidad Iberoamericana Puebla. Recuperado de https://n9.cl/nez7 
Universidad Nacional Experimental Francisco de Miranda (UNEFM). Santa Ana de Coro. Venezuela

Bayron Damián Loja-Gutama; Darwin Gabriel García-Herrera; Cristian Andrés Erazo-Álvarez; Juan Carlos Erazo-Álvarez

Phillips, B. (2017). Student-Produced Podcasts in Language Learning - Exploring Student [Podcasts sobre el aprendizaje de idiomas producidos por estudiantes: estudiante explorador]. IAFOR Journal of Education, 5(3), 157-171.

Robles, H. (2010). Experiencia de Podcasting en la Enseñanza de una Segunda Lengua. Dialnet(14), 142-149. Recuperado de https://n9.cl/63507

Ruiz, E. (2017). Aprendizaje electrónico móvil (PODCAST) en la comprensión auditiva del ingles de los estudiantes. [Mobile electronic learning (PODCAST) in the listening comprehension of the English of the studentes]. Quito: Universidad Central del Ecuador. Recuperado de https://n9.cl/ioawh

Saborío, S. (2018). Podcasting: Una herramienta de comunicación en el entorno virtual. [Podcasting: A communication the virtual environment]. Innovaciones, 20(29). doi:https://doi.org/10.22458/ie.v20i29.2254

Sigüenza , J., Montánchez , M., \& Palta, N. (05 de 2018). La tutoría académica y la herramienta web 2.0: podcast, en la educación superior. [Academic tutoring and the web tool 2.0: podcast, in higher education]. Killkana Sociales, 39-46. doi:https://doi.org/10.26871/killkana social.v2i2.297

Solano, I., \& Sanchez, M. (2010). Aprendiendo en cualquier lugar: El podcast educativo. [Learning Anywhere: The Educational Podcast.]. Pixel-Bit.(36), 125-139.

Suárez, P., Vélez, M., \& Londoño, D. (2018). Las herramientas y recursos digitales para mejorar los niveles de literacidad y el rendimiento académico de los estudiantes de primaria. [The tools and digital resources to improve the literacy levels and academic performance of elementary students]. Universidad Católica del Norte, 184-198.

UNESCO. (2013). Unesco. Recuperado el 09 de 09 de 2020, de Aprendizaje Móvil: https://n9.cl/okii 